\title{
Prevalence of zoonotic and other gastrointestinal parasites in police and house dogs in Alexandria, Egypt
}

\author{
W. M. Ahmed ${ }^{1}$, W. M. Mousa ${ }^{2}$, S. M. Aboelhadid ${ }^{1}$ and M. M. Tawfik ${ }^{3}$ \\ 1. Parasitology Department, Faculty of Veterinary Medicine, Beni-Suef University, Beni-Suef, Egypt. \\ 2. Parasitology Department, Faculty of Veterinary Medicine, Cairo University, Giza, Egypt. \\ 3. Department of Training, Security and Guard Dogs, Police Academy Alexandria, Egypt. \\ Corresponding author: W. M. Ahmed, email: wmarafa@yahoo.com, waleed.mahmoud@vet.bsu.edu.eg \\ Fax. + 202 0822327982, Cell phone: + 20201128744418 \\ Received: 25-02-2014, Revised: 28-03-2014, Accepted: 04-04-2014, Published online: 02-05-2014
}

doi: $10.14202 /$ vetworld.2014.275-280

How to cite this article: Ahmed WM, Mousa WM, Aboelhadid SM and Tawfik MM (2014) Prevalence of zoonotic and other gastrointestinal parasites in police and house dogs in Alexandria, Egypt, Veterinary World 7(5): 275-280.

\begin{abstract}
Aim: This work estimates the gastrointestinal parasites of police and house dogs in Egypt with reference to its zoonotic risk.

Materials and Methods: Fecal samples of 180 from police and house dogs were collected and then examined by different flotation and sedimentation techniques.

Results: Prevalence of gastrointestinal parasites in police dogs was $7.5 \%$ and, in house dogs was $40 \%$. Fecal examination of 120 police dogs revealed Toxocara canis eggs in 0.8\%, Ancylsotoma caninum 1.7\%, Trichuris vulpis $0.8 \%$, Cystoisospora canis $4.2 \%$ and Giardia species $1.7 \%$. Examination of 60 house dogs revealed Toxocara canis eggs in 5\% and Toxoascaris leonina $1.7 \%$, Cystoisospora canis 3.3\%, Giardia species 31.7\%, Entamoeba histolytica 18.3\% and Cryptosporidium spp. $1.7 \%$. Furthermore, age, sex, uncooked feed and communal housing revealed significant enteric parasite $(\mathrm{P}<0.05)$. Moreover, the zoonotic risks of police and house dogs were T. canis, A. caninum, T. vulpis, Giardia species, E. Histolytica and Cryptosporidium species. The puppies are representing the most zoonotic risk.
\end{abstract}

Conclusion: In spite of, hygienic measures, regular deworming and high quality feeding of police and house dogs, a range of different parasites were recorded in this work. Parasitic zoonosis from police and house dogs has to be considered, especially for dog trainers and owners.

Keywords: gastrointestinal parasites, house dogs, police dogs, zoonotic risk.

\section{Introduction}

The dog was the first domesticated animal. Dogs perform many roles for people such as hunting, pulling loads protection, assisting police, military work, companionship and more recently aiding handicapped individuals. This impact on human society has given them the nickname "Man's Best Friend" in the western world. The use of dogs in the Egyptian police began early last century, when their ability to distinguish smells that cannot be detected by humans was realized. In many parts of the world, the intestinal parasites of dogs receive considerable attention because dogs serve as reservoirs, carriers and transmitters of several pathogens, including parasites, which are considered zoonotic and number of them are of significant public health concern $[1,2]$. Nowadays, dogs are living with humans in houses all over the world. Children are at special risk of infection because of their closer direct and indirect contact to dogs as compared to adults.

The most common enteric parasites of dogs are Toxocara canis, Ancylostoma caninum, Taenia hydatigena, Echinicoccus spp., Dipylidium caninum, Trichuris vulpis, Giardia spp., Cryptosporidium spp. and Cystoisospora canis $[3,4]$.

Copyright: The authors. This article is an open access article licensed under the terms of the Creative Commons Attribution License (http://creativecommons.org/licenses/by/2.0) which permits unrestricted use, distribution and reproduction in any medium, provided the work is properly cited.
Dogs are frequently infected by intestinal parasites. Moreover, several canine intestinal parasites are zoonotic and are considered important to public health. Although dogs are often considered family members by their owners, it is important to emphasize that they may be vectors of intestinal parasites. Although internal parasites cause mostly chronic asymptomatic types of infections, they can cause clinical disease in dogs depending on the burden and pathogenicity of the parasites. In addition, dogs are definitive hosts for a range of parasites with heterogeneous life cycles for which herbivorous and omnivorous animals serve as intermediate hosts such as Echinococcus spp. and Neospora spp.

The canine intestinal parasites have an oral-fecal transmission cycle and a major component for the spread of these parasites is the shedding of oocysts or cysts and eggs or larvae into the environment $[4,5]$. The transmission of zoonotic agents could be through indirect contact with animal secretions and excreta, infected water and food, or through direct contact with the animal [6].

The target of this study was to determine the prevalence of zoonotic and other gastrointestinal parasites (helminthes and protozoa) in both police and house dogs in Alexandria, Egypt.

\section{Materials and Methods}

Ethical approval: Not necessary. 
Table-1: Prevalence of enteric parasite species detected in police and house dogs feces.

\begin{tabular}{|c|c|c|c|c|c|c|c|}
\hline \multirow[t]{2}{*}{ Parasite } & \multicolumn{2}{|c|}{ Police dogs $(n=120)$} & \multicolumn{2}{|c|}{ House dogs $(n=60)$} & \multirow[t]{2}{*}{ Chi-square } & \multirow[t]{2}{*}{$P$ - value } & \multirow[t]{2}{*}{ Zoonotic potential } \\
\hline & $\begin{array}{c}\text { No. } \\
\text { positive }\end{array}$ & $\begin{array}{c}\text { Prevalence } \\
(\%)\end{array}$ & $\begin{array}{c}\text { No. } \\
\text { positive }\end{array}$ & $\begin{array}{c}\text { Prevalence } \\
(\%)\end{array}$ & & & \\
\hline Ancylostoma caninum & 2 & 1.7 & 0 & 0 & 1.0112 & 0.314607 & $\begin{array}{l}\text { Cutaneous larva migrans and } \\
\text { eosinophilic enteritis }\end{array}$ \\
\hline Toxocara canis & 1 & 0.8 & 3 & 5 & 3.196 & 0.073818 & $\begin{array}{l}\text { Visceral and ocular larva } \\
\text { migrans }\end{array}$ \\
\hline Toxoscaris leonina & 0 & 0 & 1 & 1.7 & 2.0112 & 0.156145 & None \\
\hline Trichuris vulpis & 1 & 0.8 & 0 & 0 & 0.5028 & 0.478275 & Duodenal ulcer disease \\
\hline Cystoisospora canis & 5 & 4.2 & 2 & 3.3 & 0.0743 & 0.785149 & None \\
\hline Giardia species & 2 & 1.7 & 19 & 31.7 & 34.9326 & $0^{*}$ & Giardiasis \\
\hline Entamoeba histolytica & 0 & 0 & 11 & 18.3 & 23.432 & $<0.05^{*}$ & Entameobiasis \\
\hline Cryptosporidium species & 0 & 0 & 1 & 1.7 & 2.0112 & 0.156145 & Cryptosporidiosis \\
\hline
\end{tabular}

* mean there is a significant difference between house and police dogs at $\mathrm{P}<0.05$. Some animals have single parasitic infection (Mono infection) and others have mixed infection (hetero infection).

Study area: This study was conducted during the period from January 2012 to January 2013 on police dogs from Department of Training Security and Guard Dogs, Police Academy and house dogs in Alexandria, the second largest city and the second largest metropolitan area in Egypt after Greater Cairo.

Animals: The study animals included 180 dogs divided into two groups:

A- Police dogs: One hundred twenty police dogs (112 males and 8 females) were of exotic(pure) breeds. They were categorized according to age into three categories from 0 to 6 months (12), from 6 months to 1 year (12) and older than 1 year (96). Each animal was housed in a single cage and fed on dry food. All animals were subjected to regular deworming protocol. Puppies were dewormed at 2 weeks of age and then repeated at 5 weeks, 8 weeks, 12 weeks and 16 weeks with fenbendazole (granules 22.2\%). Adult dogs receive their deworming (Fenbendazole) twice per year. In pregnant bitches, Fenbendazole used from day 40 of pregnancy to 2 days post whelping.

B- House dogs: Sixty house dogs (42 males and 19 females), 16 were of local (mixed) Egyptian breeds and 44 of pure exotic breeds. They were categorized according to age into three categories from 0 to 6 months (9), from 6 months to 1 year (11) and older than 1 year (40). All animals were group-housed (more than one dog per house) and fed on uncooked food.

Fecal samples collection: Fresh fecal samples were collected by server (police dogs) or by owner (house dogs) from the ground in the morning immediately after voiding by dogs and individually labelled in plastic containers. Each sample represented one animal and was collected from both asymptomatic and symptomatic dogs. Formalin $(10 \%)$ was used as preservative for the samples. The age, gender, history of anthlemintic usage, housing and feeding systems were recorded for each dog.

Examination techniques: The fecal samples were examined macroscopically for adult nematodes and tapeworm proglottids. Each sample was subjected for examination by centrifugal fecal floatation technique using different solutions (saturated salt solution, zinc sulphate and Sheather's solution) and fecal sedimentation technique [7]. Furthermore, formol ether sedimentation technique [8] was applied for each sample. Iodine solution was used to facilitate protozoan and cyst identification. The modified ZiehlNeelsen (MZN) staining technique is used to detect Cryptosporidium spp.oocysts in the feces [9]. Parasites were identified on the basis of eggs, oocysts or cysts color, shape and contents $[7,10]$.

Statistical analysis: Raw data were entered into a Microsoft Excel spreadsheet and descriptive statistics were used to summarise the data. The prevalence was calculated for all data as the number of infected individuals divided by the number of individuals examined and multiplied by 100 to express in percentage. Chi square was used to assess the association of risk factors on the prevalence of parasites. All statistical analyses were performed using the "SPSS" statistical software. The significance level was $\mathrm{P}<0.05$ [11].

\section{Results}

Prevalence of enteric parasites in police and house dogs: The overall prevalence of enteric parasites infection by fecal examination was $18.3 \%$ (33/180) in all examined dogs. The prevalence of the infection in police dogs was 7.5\% (9/120 animals) and, in house dogs, was $40 \%$ animals (24 of 60 ). The recovered helminth eggs were $A$. caninum, T. canis, T. leonina and Trichuris vulpis. In addition, the recorded protozoa were Cystoisospora canis, Giardia species, Entamoeba histolytica and Cryptosporidium spp oocyts (Table-1, Plates-1, 2 and 3, Figure-1 and 2).

Risk factors and enteric parasite infections in police and house dogs: The risk factors are age, sex, breed, feeding and housing systems. According to age, puppies (under 6 months) showed significant enteric parasite prevalence $66.6 \%$ ( $\mathrm{P}<0.000)$. In addition, females showed significant enteric parasite prevalence $33.3 \%(\mathrm{P}<0.05)$ compared with males $(15.7 \%)$. Other risk factors like, the uncooked feeding and the communal housing, revealed significant enteric parasite prevalence $40 \%(\mathrm{P}<0.000)$ in comparison to dry feed or individual housing systems $(7.50 \%)$ (Table- 2$)$. 


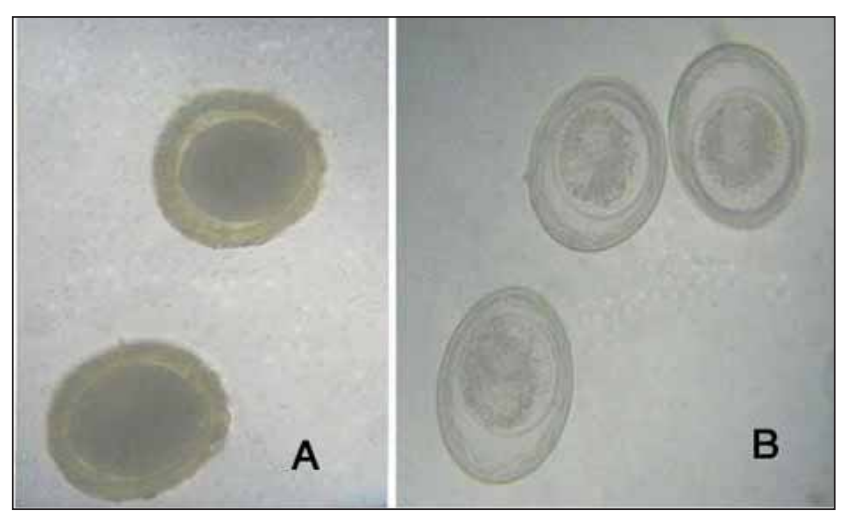

Plate-1: A. Toxocara canis eggs ( $80 \times 80 \mathrm{um})$. B. Toxoascaris leonine eggs $(72.5 \times 80 \mathrm{um})$. The two species can be differentiated by egg shell morphology: egg shell of $T$. canis is granular and that of $T$. leonina is lamellated.
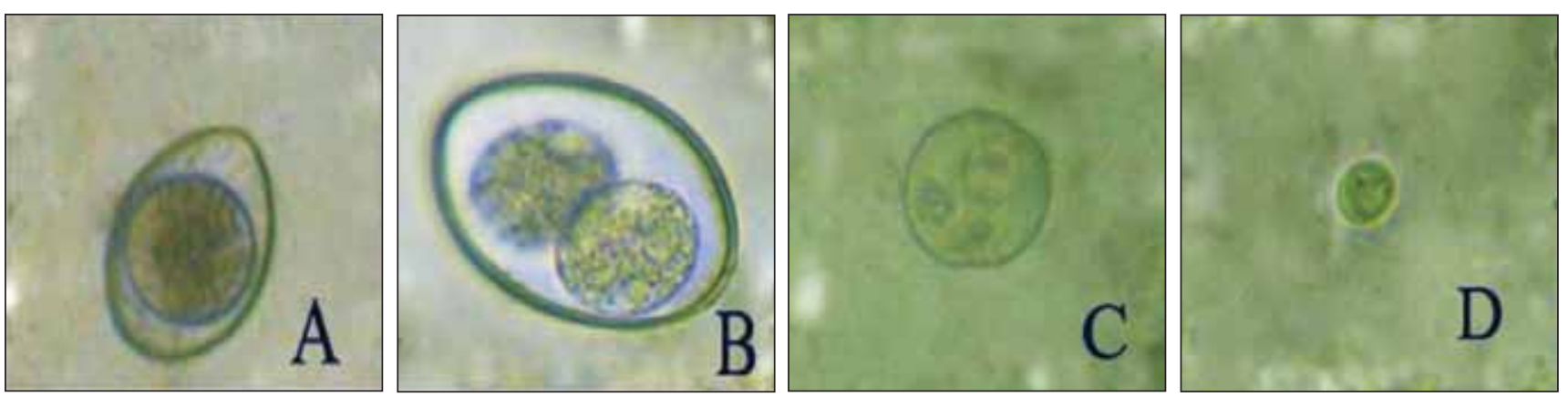

Plate-3: A. Cystoisospora canis sporulated oocyst (22.5-25×30um). B. Cystoisospora canis sporulated oocyst (2 sporocyst). C. Entamoeba histolytica cyst ( $12 \times 15$ um). Mature cyst has 4 nuclei; immature cyst has 1 or 2 nuclei. D. Giardia spp cyst ( $9 \times$ 7um). Cysts are elliptical with a thin, smooth cyst wall and contain two to four nuclei.

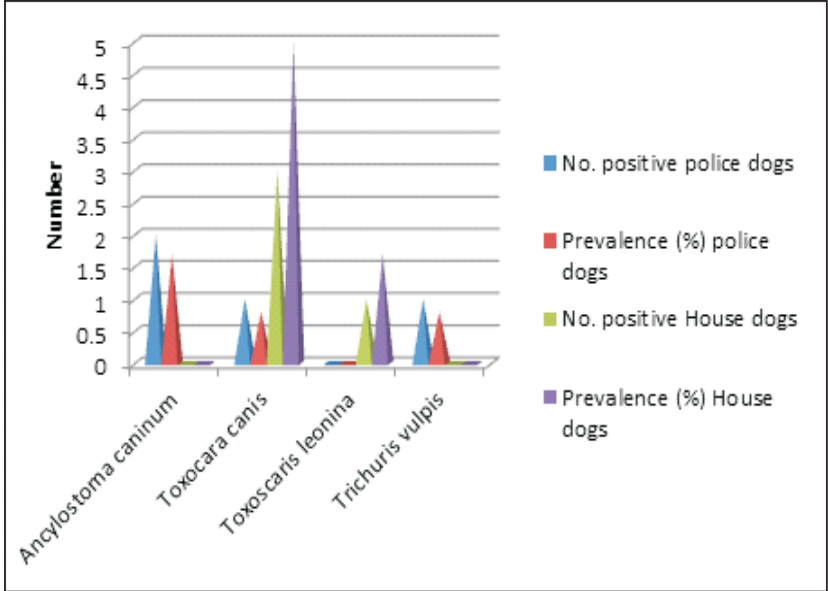

Figure-1: Prevalence of enteric helminth species detected in police and house dogs.

Regarding age and the parasite species infecting dogs, the identified helminth eggs (A. caninum, $T$. canis, T. leonina and Trichuris vulpis) were significantly prevalent in puppies $(\mathrm{P}<0.05)$. In the same way, Cystoisospora canis oocyts were significantly prevalent in puppies $(\mathrm{P}<0.000)$. On the other hand, age was not a factor for enteric parasite prevalence in relation to Giardia species, E. histolytica and Cryptosporidium species (Table-3).

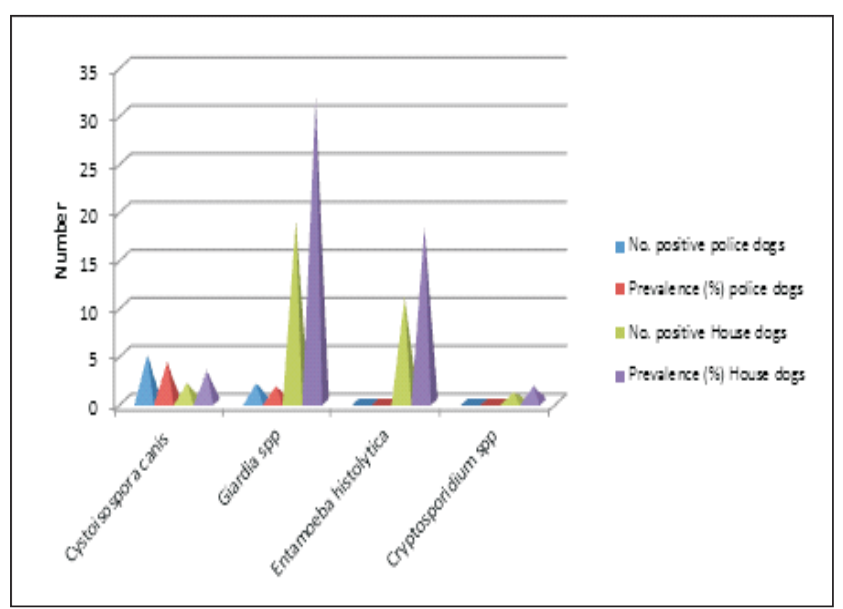

Figure-2: Prevalence of enteric protozoa species detected in police and house dogs.

The zoonotic impact of the enteric parasites species in police and house dogs: The enteric parasites recorded in this study showed an impact of zoonosis and hazard to public health. These parasites are A. caninum, $T$. canis, T. vulpis and Giardia species in police dogs. In addition, T. canis, Giardia species, E. histolytica and Cryptosporidium species were of zoonotic potential in house dogs. Moreover, Giardia spp. and E. histolytica were found to be the common parasites which could be 
Table-2: The risk factors effect on enteric parasites affection in police and house dogs.

\begin{tabular}{|c|c|c|c|c|c|c|}
\hline Risk factors & Categories & No. examined & No. positive & Prevalence (\%) & Chi-square & P-value \\
\hline \multirow[t]{3}{*}{ Age } & Under 6months & 21 & 14 & 66.6 & & \\
\hline & 6-12months & 23 & 2 & 8.7 & & \\
\hline & $>1$ year & 136 & 17 & 12.5 & 37.3 & 0.000 \\
\hline \multirow[t]{2}{*}{ Sex } & Male & 153 & 24 & 15.7 & & \\
\hline & Female & 27 & 9 & 33.3 & 4.77 & 0.02 \\
\hline \multirow[t]{2}{*}{ Breed } & Local & 16 & 4 & 25 & & \\
\hline & Exotic & 164 & 29 & 17.7 & 0.521 & 0.470 \\
\hline \multirow[t]{2}{*}{ Feeding } & Dry & 120 & 9 & 7.5 & & \\
\hline & Uncooked & 60 & 24 & 40 & 37.006 & 0.000 \\
\hline \multirow[t]{2}{*}{ Housing } & Individual & 120 & 9 & 7.5 & & \\
\hline & Communal & 60 & 24 & 40 & 37.006 & 0.000 \\
\hline
\end{tabular}

Table-3: Prevalence of parasite species in relation to age in police and house dogs.

\begin{tabular}{|c|c|c|c|c|c|c|}
\hline Risk factors & Categories & No. examined & No. positive & Prevalence (\%) & Chi-square & P-value \\
\hline \multirow[t]{3}{*}{ A. Caninum } & Under 6months & 21 & 2 & 9.5 & & \\
\hline & 6-12months & 23 & 0 & 0 & & \\
\hline & >1year & 136 & 0 & 0 & 15.313 & .000 \\
\hline \multirow[t]{3}{*}{ T. canis } & Under 6months & 21 & 1 & 9.5 & & \\
\hline & 6-12month & 23 & 1 & 4.3 & & \\
\hline & >1year & 136 & 1 & 0.7 & 7.015 & .030 \\
\hline \multirow[t]{3}{*}{ T. leonina } & Under 6months & 21 & 1 & 4.8 & & \\
\hline & $6-12 \mathrm{~ms}$ & 23 & 0 & 0 & & \\
\hline & $>1$ year & 136 & 0 & 0 & 7.614 & .022 \\
\hline \multirow{3}{*}{ Trichuris vulpis } & Under 6months & 21 & 1 & 4.8 & & \\
\hline & 6-12months & 23 & 0 & 0 & & \\
\hline & >1year & 136 & 0 & 0 & 7.614 & .022 \\
\hline \multirow[t]{3}{*}{ Cystoisospora canis } & Under 6months & 21 & 7 & 33.3 & & \\
\hline & 6-12months & 23 & 0 & 0 & & \\
\hline & >1year & 136 & 0 & 0 & 55.145 & .000 \\
\hline \multirow[t]{3}{*}{ Giardia spp } & Under 6months & 21 & 3 & 14.3 & & \\
\hline & $6-12$ months & 23 & 2 & 8.7 & & \\
\hline & >1year & 136 & 16 & 11.8 & 0.338 & .844 \\
\hline \multirow[t]{3}{*}{ Entamoeba histolytica } & Under 6months & 21 & 3 & 14.3 & & \\
\hline & 6-12months & 23 & 1 & 4.3 & & \\
\hline & >1year & 136 & 7 & 5.1 & 2.791 & .248 \\
\hline \multirow[t]{3}{*}{ Cryptosporidium spp. } & Under 6months & 21 & 0 & 0 & & \\
\hline & 6-12months & 23 & 1 & 0 & & \\
\hline & >1year & 136 & 0 & 0.70 .7 & 0.325 & .850 \\
\hline
\end{tabular}

of zoonotic importance coming from house dogs (Table1). The puppies were the suitable and the most susceptible for these parasites except for Cryptosporidium spp. which was recorded in aged dogs (over one year)(Table-3).

\section{Discussion}

Ongoing levels of pet ownership all over the world raises the importance of dogs. In addition, commercial and official use of dogs in international showing, security and in the military increases the demand and attention to these animals. One of the most common ailments of dogs all over the world is the parasitic infection especially the intestinal ones. Moreover, several canine parasites have a zoonotic importance and represent a public health hazard.

In this work a total number of 180 fecal samples of police and house dogs were examined by different techniques. However, the hygienic measures, regular deworming and high quality feeding of police and house dogs, different parasites were recorded in this work; the total percentages of parasites infection were $(18.3 \%)$. This finding is supported by Senlik et al. [12], who clarified that, even with a relatively high level of care, including veterinary attention; military dogs frequently harbour intestinal nematodes. In addition, they recorded a percentage of $30.4 \%$ of the examined dogs were found to be infected with one or two nematode species.

According to risk factors, animals puppies under 6 months of age showed the significant enteric parasite prevalence to be $66.6 \%(\mathrm{P}<0.000)$ in a comparison with the other age groups. The effect of risk factors enforced by Abere et al. [11], who estimated the parasites prevalence, was strongly associated with age. Also the same author showed that the overall prevalence of parasites was significantly higher in young dogs $(<1$ year) than adults [11]. This was partially due to parasite specific immunity usually acquired with age or probably as a consequence of single or repeated exposures [13].

Concerning the sex as a risk factor, females showed significant enteric parasite prevalence 33.3\% at $(\mathrm{P}<0.05)$ than male dogs $(15.7 \%)$. Since, females of police and house dogs included in the comparison with the males, other risk factors such as age, uncooked food and communal housing may interfere with the sex effect. A study in Nigeria indicated that female dogs were more likely to contract intestinal nematodes than male dogs [14]. On the other side, Zelalem et al. [15], found that, prevalence of gastrointestinal helminthes was higher in male dogs $(79.2 \%)$ than female $(76.8 \%)$ dogs [15].

Breed was not found statistically significant as a 
risk factor for the prevalence of gastrointestinal helminthes in local, cross and exotic breeds of dogs. This report agrees with the previous study of Swai et al. [16] in Tanzania. This indicates that all breeds have equal chance of acquiring the infection if they are exposed to infected materials. On the contrary, prevalence of helminthes in exotic-breed dogs were higher $(81.3 \%)$ than local-breed dogs (76.6\%) [15]. Uncooked feeding revealed significant enteric parasite prevalence $40 \%$ (P $<0.000$ ). Feeding as a risk factor go parallel with study of Zelalem, G. and Mekonnen [15] who clarified that, prevalence of gastrointestinal helminthes was highest in dogs that were fed raw (93.7\%) followed by dogs that feed mixed (90.7\%) and cooked (37.5\%) feed items. Statistical analysis of the data showed significant difference $(\mathrm{P}<0.05)$ in prevalence of helminthes between feed types. The highest prevalence of gastrointestinal helminthes in dogs that were fed raw is clarified by the fact that cooking can kill or inactivate infective eggs or cysts of gastrointestinal helminthes which could be transferred among dogs via feed [15]. Also, communal housing, showed significant enteric parasite prevalence $40 \%(\mathrm{P}<0.000)$. These findings were augmented by [11] who showed that feeding management had a significant influence in the prevalence of gastrointestinal helminth infections. Dogs which receive great care from their owners had lower incidence of intestinal helminths than dogs lacking such privileges.

It is of interest to mention; dogs are coprophagic and will eat not only dog feces but feces of many other species as well. This brings up the possibility that the amoeba cysts, Cryptosporidium and Eimeria oocysts were from ingested feces of other species and may just have been passing though the dog, not an actual infection. The lack of these infections in police dogs which are better managed and not allowed to roam would support this.

The investigation of the zoonotic risk revealed that, T. canis, A. caninum, T. vulpis, Giardia spp and Cryptosporidium spp. represent zoonotic risk factors for the dog trainers and owners. The zoonotic potentiality of these parasites is supported by [17]. T. canis was detected in both police and house dogs 0.8 and 5\% respectively. It is worth mentioning that, puppies represent a higher zoonotic risk than adults at $\mathrm{p}<0.05$. T. canis infections recorded in young dogs is in agreement with its route of migration (lactogenic and transplacental ways). This finding is supported by Senlik, et al. [12] who recorded that, $T$. canis infection of Turkish military dogs was $(13.3 \%)$. Also they recorded that, $T$. canis infections were more frequently seen in puppies (0-6 months old). Moreover, Overgaauw, P.A. and Boersema, J.H. [18] recorded T. canis $21 \%$ in adult dogs and $48 \%$ in puppies. Prevalence of T. canis was higher in young (48.9\%) dogs than in adults $(22.8 \%)$ [15]. Human toxocariasis (toxocaral larva migrans) has been reported to be the most common zoonotic parasitic infection acquired from pets, as people become infected when they accidentally ingest larvae. This is more common in children who often practice pica [19]. A. caninum was found in police dogs (1.7\%). The larvae of hookworms (Ancylostoma and Uncinaria) can penetrate the skin of humans as well as the relevant canine or feline host. According to the present study, the server of police dogs is under risk of infection by this parasite through cleaning of dog cages in an unhygienic manner. When the larvae migrate under the skin, progressive linear eruptions occur, resulting in cutaneous larva migrans or creeping eruption. This condition is more common in areas of higher humidity (tropical and subtropical regions), particularly in people who have to crawl beneath buildings, sunbathers who sun bake in areas contaminated by hookworm larvae, or people who walk around barefooted [20]. Daily removal of faeces from the environment will reduce the chance of infection in both pets and their owners.

It is of interest to note about the zoonotic importance of $T$. vulpis. The risk of $T$. vulpis was supported by Dunn, et al. [21] who recorded a case of human infection with the whipworm of dogs, T. vulpis, in a woman with duodenal ulcer disease, chronic diarrhea, and close contact with dogs. Morphologically, T. vulpis ova resemble those of the human whipworm (T. trichiura) but are nearly twice their size. Few cases of presumed human infection by T. vulpis had been described [22]. On the other side, Donato [23] viewed that clear data still need to be provided to definitively add this parasite to the causes of human intestinal infections. Giardia spp. was recorded in police $(1.7 \%)$ and house dogs $(31.7 \%)$. This high prevalence of Giardia among the Shelterd Dogs is recoded by [24]. Epidemiological evidence would suggest that humans are likely to be the main reservoir of human giardiasis and it is likely that direct person-toperson transmission is more important than zoonotic transmission. However, dogs and cats can carry strains of Giardia which are potentially infective to humans, and therefore, the zoonotic potential must be considered, especially for immunocompromised people [17]. The zoonotic potential of canine Giardia remains largely an unresolved issue [1].

Zoonotic potentiality of Cryptosporidium spp. should be considered which is supported by Sargent et al. [25], who clarified that Cryptosporidium had also been detected in dogs and cats, and these animals may represent potential sources of infection for humans.

\section{Conclusion}

It could be concluded that, the parasitic infection rate was higher in house dogs $(40 \%)$ than police dogs (7.5\%). Puppies, females, dogs that are fed on raw stuff and dogs in communal housing are the more susceptible to enteric parasites infection. It is of interest to note that all the estimated parasites either helminths or protozoa have a direct life cycle. These animals were housed under captivity so the chances for harbouring cedstodes or trematodes, which require an intermediate host, were limited. Inspite of, hygienic measures, 
regular deworming and high quality feeding of police and house dogs, a range of different parasites were recorded in this work. Parasitic zoonosis from police and house dogs has to be considered, especially for dog trainers and owners. Puppies represent the highest zoonotic risk for these zoonotic parasites specially Giardia species and E. histolytica.

\section{Authors' contributions}

WMM, SMA and WMA: Planned the study design. MMT and WMA: Sample collection and examination. WMA and SMA: Statistical analysis, draft and revision of the manuscript. All authors read and approved the final manuscript.

\section{Acknowledgments}

The authors are thankful to Fiona Tegert, research assistant at Monash University, Australia for her help in manuscript language revision. This work was funded by Faculty of Veterinary Medicine, Beni-Suef University, Egypt and Department of Training Security and Guard Dogs, Police Academy Alexandria, Egypt.

\section{Competing interests}

The authors declare that they have no competing interests.

\section{References}

1. Traub, R.J., Robertson I.D., Irwin P.J., Mencke N. and Thompson R.C. (2005) Canine gastrointestinal parasitic zoonoses in India. Trends Parasitol., 21: 42-48.

2. Gracenea M., Gómez M.S. and Torres J. (2009) Prevalence of intestinal parasites in shelter dogs and cats in the metropolitan area of Barcelona (Spain). Acta Parasitol., 54:73-77.

3. Palmer C.S., Thampson R.C.A., Traub R.J., Ress R. and Robertson R.D. (2008) National study of the gastrointestinal parasites of dogs and cats in Australia. Vet. Parasitol., 151: 181-190.

4. Khante G.S., Khan L.A., Bodkhe A.M., Suryawanshi P.R., Majed M.A., Suradkar U.S. and Gaikwad S.S. (2009) Epidemiological survey of Gastro-intestinal parasites of non-descript dogs in Nagpur city. Vet. World, 2 (1): 22-23.

5. Claerebout E., Casaert S., Dalemans A.C., De Wilde, N., Levecke B., Vercruysse J. and Geurden T. (2009) Giardia and other intestinal parasites in different dog's populations in Northern Belgium. Vet. Parasitol., 161:41-46.

6. Bugg R.J., Robertson I.D., Eliot A.D. and Thompson R.C.A. (1999) Gastrointestinal parasites of urban dogs in Perth, Western Australia. Vet. J.157(3): 295-801.

7. Zajac, A.M. and Conboy, G.A. (2012) Veterinary clinical parasitology 8th edition, John Wiley \& Sons. p40-87.

8. Lee, J., Kang, S., Kim, N., Lee, C., Ahn, K., Kwon, H., Park, C. and Kim, S. (2010) Investigation of helminths and protozoans infecting old world monkeys: captive vervet, cynomolgus, and rhesus monkeys. Korean J. Vet. Res., 50 (4): $273-277$.

9. Henriksen, S.A. and Pohlenz, J.F.L. (1981) Staining of Cryptosporidia by a modified Ziehl-Neelsen technique. Acta Vet. Scand., 22 : 594-596.

10. Soulsby, E.J.L. (1982) Helminths, arthropods, and protozoa of domesticated animals. $7^{\text {th }}$ Ed. Lea \&Febiger, Philadelphia.

11. Abere, T., Bogale, B. and Melaku, A. (2013) Gastrointestinal helminth parasites of pet and stray dogs as a potential risk for human health in Bahir Dar town, north-western Ethiopia, Vet. World, 6(7): 388-392.

12. Senlik, B., Cirak, V.Y. and Karabacak, A. (2006) Intestinal nematode infections in Turkish military dogs with special reference to Toxocara canis. J. Helminthol., 80 (3): 299-303.

13. Ramírez-Barrios, R.A., Barboza-Menab, G., Muñoza, J., AnguloCubillána, F., Hernándezb, E., Gonzálezb, F. and Escalona, F. (2004) Prevalence of intestinal parasites in dogs under veterinary care in Maracaibo, Venezuela. Vet. Parasitol., 121 (1-2): 11-20.

14. Davoust, B., Normand, T., Bourry, O., Dang, H., Leroy, E. and Bourdoiseau, G. (2008) Epidemiological survey on gastrointestinal and blood-borne helminths of dogs in north east Gabon. Onderstepoort J Vet. Res., 75 : 359-364.

15. Zelalem, G. and Mekonnen, A. (2012) Prevalence of Gastrointestinal Helminthes among Dogs in Bahir Dar Town, Ethiopia. World Appl. Sci. J., 19 (5): 595-601.

16. Swai, E.S., Kaaya, E.J., Mshanga, D.A. and Mbise, E.W. (2010) A Survey on Gastro-intestinal Parasites of nondescript dogs in and around Arusha Municipality, Tanzania. Int. J. Anim. Vet. Adv. 3 (2): 63-67.

17. Robertson, D. and Thompson, R. (2002) Enteric parasitic zoonoses of domesticated dogs and cats, Microbes Infect., 4: $867-873$

18. Overgaauw, P.A. and Boersema, J.H. (1998) Nematode infections in dog breeding kennels in the Netherlands, with special reference to Toxocara. Vet. Q., 20 (1): 12-5.

19. Overgaauw, P. (1997) Aspects of Toxocara epidemiology, toxocarosis in dogs and cats. Crit Rev. Microbiol., 23: 233-251.

20. Walker, N.I., Croese, J., Clouston, A.D., Parry, M., Loukas, A. and Prociv, P. (1995) Eosinophilic enteritis in northeastern Australia-Pathology, association with Ancylostoma caninum and implications. Am. J. Surg. Path. 19:328-337.

21. Dunn, J., Columbus, S., Aldeen, W., Davis, M. and Carroll, K. (2002) Trichuris vulpis recovered from a Patient with Chronic Diarrhea and Five Dogs. J. Clin. Microbiol, 2703-2704.

22. Kirkova, Z., Georgiev, D. and Raychev, E. (2006) Study on the prevalence of trichurosis in different categories of dogs and wild carnivores. Bulgarian J. Vet. Med., 9:141-147.

23. Donato T. (2011) Are we paying too much attention to cardio-pulmonary nematodes and neglecting old-fashioned worms like Trichuris vulpis? Parasites \& Vectors 8;4:32.

24. Ortuño A,.Castellà J. (2011) Intestinal Parasites in Shelter Dogs and Risk Factors Associated withthe Facility and its Management. Isr J Vet Med., Vol. 66 (3).

25. Sargent K.D., Morgan U.M., Elliot A.D., Thompson R.C.A. (1998) Morphological and genetic characterisation of Cryptosporidium oocysts from domestic cats. Vet Parasitol 77:221-227. 